\title{
Ship Collision Avoidance Path Planning Strategy Based on Quantum Bacterial Foraging Algorithm
}

\author{
Liu Hongdan ${ }^{1, a}$, Liusheng ${ }^{2, b}$, Zhanglanyong ${ }^{3,}$ \\ ${ }^{1}$ College of Automation, Harbin Engineering University, Harbin 150001, China; \\ ${ }^{2}$ College of Automation, Harbin Engineering University, Harbin 150001, China; \\ ${ }^{3}$ College of Automation, Harbin Engineering University, Harbin 150001, China; \\ aliuhongdan131@126.com, 'bliusheng@hrbeu.edu.cn, 'zhanglanyong@hrbeu.edu.cn
}

Keywords: Ship Collision Avoidance; Path Planning; Quantum Algorithm; Bacterial Foraging Algorithm; Optimization Parameters

\begin{abstract}
In order to give an early warning for collision situation in marine traffic, to provide a collision avoidance decision, to relieve the workload of sailors, and to guarantee the safety of ship navigation, quantum bacteria foraging optimization algorithm is used to plan the dynamic collision avoidance process of ships by setting the shortest sailing distance in the collision avoidance process of own ship as the objective function according to COLREGS (Convention on the International Regulations for Preventing Collisions at Sea) and relevant knowledge in ship safety field. Meanwhile, the optimized avoidance angle, sailing time of resumption, resumption opportunity, and resumption angle in ship collision avoidance path planning are provided. Finally, effectiveness and superiority of this strategy are verified.
\end{abstract}

\section{Introduction}

Ship collision avoidance system is a complicated decision-making process, as shown in Fig. 1. Covering various dynamic or static data, certain or uncertain information, complex mathematical calculation, and plentiful theoretical derivations, it requires a large amount of disciplinary knowledge including navigation, computer science and other fields. Moreover, it also involves different techniques like data collection, data preprocessing, division of encounter situation, calculation of collision risk degree, judgment of encounter conditions, selection of collision avoidance mode, optimization of collision avoidance action, establishment of navigation restoration decision, and multi-object collision avoidance. Therefore, determination for safe collision avoidance path of ships is a multi-standard and nonlinear path planning issue. Thereby, research scholars begin to apply various kinds of artificial intelligence technology to ship collision avoidance field[1][2][3][4], so as to realize a balance between navigation" safety "and "economy". This means that the adopted ship collision avoidance mechanism should not only maintain relevant risk assessment and collision avoidance measures in navigation, but also consider deviation from the original path.

Based on bacterial foraging algorithm, this paper self-adaptively adjusts the movement step length in chemotactic operation and sets up a quantized potential energy well model in copy operation. Copy operation is completed via Monte Carlo random sampling. In this way, the bacteria are able to search in the whole space and quantum bacterial foraging algorithm is obtained. Based on this algorithm, by combining with COLREGS and relevant knowledge in ship safety field, the optimal ship collision avoidance path is acquired. Besides, the dynamic collision avoidance process is gained through simulation experiment. 


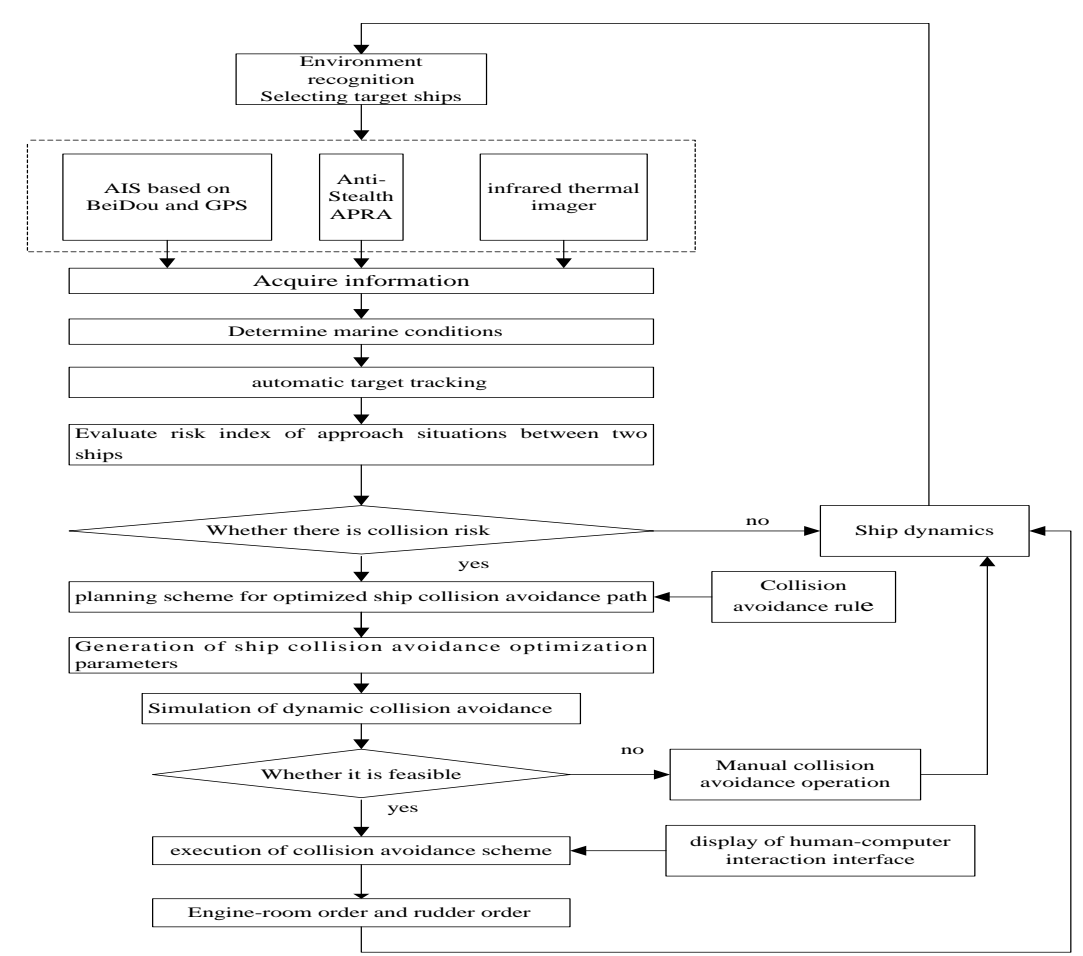

Fig.1. Implementation Process of Ship Collision Avoidance System

\section{Division OF Encounter Situation}

Collision avoidance path which is produced by ship collision avoidance decision support system must ensure that the target ship sails beyond the own ship field .It is necessary to define the encounter situations of vessels to apply with COLREG [5][6]. When two ships approach at sea, there can be several situations, including head-on (F), crossing (A, B, E), and overtaking (C, D), as shown in Figure 2.

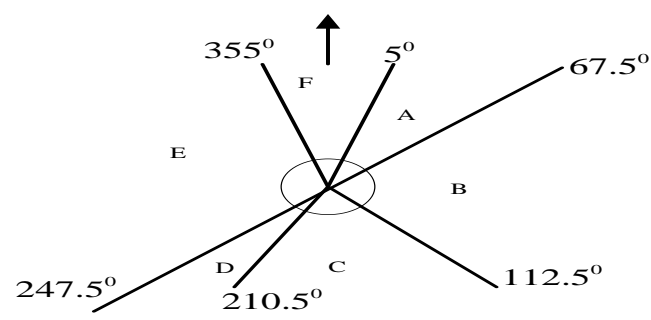

Fig.2. Division diagram for ship encounter situation

And the rule of "action to avoid collision" for each situation are showed in the following according to COLREGS rule from 13 to 17.

Rule 13 Overtaking: an overtaking vessel must keep well clear of the vessel being overtaken.

Rule 14 Head-on Situations: when two power-driven vessels are meeting head-on both must alter course to starboard so that they pass on the port side of the other.

Rule 15 Crossing Situations: when two power-driven vessels are crossing, the vessel, which has the other on the starboard side, must give way.

Rule 16 The Give-Way Vessel: the give-way vessel must take early and substantial action to keep well clear.

Rule 17 The Stand-On Vessel: the stand-on vessel may take action to avoid collision if it becomes clear that the give-way vessel is not taking appropriate action.

\section{Path planning Model of Ship Collision Avoidance}

The target ship and own ship enter the stage of navigation observation after judging the encounter situation between ships. The encounter situation of two ships is judged according to 
COLREGS, and then whether own ship is the give-way vessel will be determined. If own ship is the give-way vessel and collision risk exists between the target ship and own ship, the intelligent decision support system will execute collision avoidance path planning and recommend a safe and economic path, which can give an early warning and auxiliary decision, be used as a reference of collision avoidance scheme for sailor. And collision avoidance path planning for ships is divided into 3 stages according to different task stages.

1) Initial vigilance stage: the encounter situation between the target ship and own ship will be judged after the target ship enters the observation stage of own ship. Whether there is collision risk with the target ship and own ship is the give-way vessel will be determined. At this stage, the nearest collision avoidance turning point is decided. Meanwhile, the maintenance period for the original sailing velocity and direction can be gained. The above information can be used as warning sign at the early steering stage.

2)the stage of collision avoidance sailing: taking into account specific character of safe ship control process, characterized by great course changes in range from $20^{\circ}$ to $90^{\circ}$. Furthermore, the change of course (avoiding angle)is better over $25^{\circ}$ to make the targets ships to attention and reduction of speed is no more than 30\% in order to meet requirements of rule 8b of COLREGS.

3) Resumption stage: When decisions about resumption time and resumption action are made, the optimal resumption angle should be adopted, so as to complete the entire collision avoidance process.

\section{Ship Collision Avoidance Path Planning Analysis Strategy Based on Bacterial Foraging algorithm}

\section{Quantum bacteria foraging optimization algorithm.}

Bacterial foraging (BFO) algorithm[7][8]realizes powerful local search of the individual via continuous alternation of movement and flipping in chemotactic operation. Therefore, the chemotactic velocity affects convergence precision of the algorithm. Secondly, the scale of bacteria population is guaranteed through copy behaviors of bacteria. However, limitation of population will be caused easily and the algorithm might fall into local optimum. Finally, it comes to migration operation. Sudden changes might happen to local regions where individual bacterium lives, which might result in collective death of bacteria population in this region. Perhaps they might migrate to a new local region according to the given migration probability, and the ability of global search will be enhanced. Bacterial foraging algorithm completes optimization of individual bacterium based on nested loop of the above three steps. See Fig. 3 for the realization process of bacteria foraging optimization algorithm.

\section{Chemotactic optimization operation.}

Chemotactic movement of bacteria directly affects local convergence rate of the algorithm. By combining with the characteristic of cosine function that its slope changes slowly in the interval of $(0, \pi)$, decreases substantially in the middle, and then tends to be gentle, cosine function is used to adjust the movement step length of bacteria. This meets the dynamic search rule of bacteria population more. Bacteria distribute randomly at the initial stage of foraging and they are far away from the optimal solution. Therefore, they need to search in the foraging region with a great step length. The function value of cosine function presents a decline trend in the interval of $(0, \pi)$. Besides, its stable slope change at the beginning meets the requirement of great step length at this stage. As a result, the bacteria will not center on the initial value and move around it within a certain chemotactic period due to the small step length, and local precocity phenomenon can be overcome. After foraging for some time, the bacteria population approaches the optimal solution, so the chemotactic operation needs to transform from great step length into fine search stage. This rule matches the characteristic of cosine function that its value decreases substantially in the middle. At the end of chemotactic operation in bacterial foraging, the bacteria population is almost near the optimal solution and the slope of cosine function tends to be gentle again. At this time, the bacteria population can further enhance the optimization precision by adopting a stable and small movement 
step length. Thereby, the change rule of cosine function is added into movement step length, and the following formula is gained after improvement:

$$
C(i, j, w)=\frac{\left(C_{\max }-C_{\min }\right)}{2} \cos \left(\frac{\pi}{N_{s}} w\right)+\frac{\left(C_{\max }+C_{\min }\right)}{2}, i=1,2 \ldots, N, w=0,1, . ., N_{s}
$$

Where $C_{\max }$ and $C_{\min }$ are maximum value and minimum value of movement step length, $N_{s}$ indicates the maximum chemotactic frequency at a certain direction in chemotactic operation, and $C(i, j, w)$ denotes the movement step length of bacterium $i$ in chemotactic operation $j$ during movement $w$. Meanwhile, based on the bacterial foraging algorithm, the best objective value of each bacterium can be obtained when all bacteria have completed chemo taxis. At that point, the optimal bacterial tumbling direction and the new position for the next chemo taxis and can be optimized based on particle swarm optimization in (2) and (3)

$$
\begin{aligned}
& V=w^{*} V+C_{1} * R_{1} *\left(P_{\text {lbest }}-P\right)+C_{2} * R_{2} *\left(P_{\text {gbest }}-P\right) \\
& P(x, y)=P(x, y)+C * V
\end{aligned}
$$

Where, $V$ and $P$ is of the next the chemo taxis, $C_{1}, R_{1}, C_{2}, R_{2}$ are random parameters. the $P_{l b e s t}$ is single optimal solution, $P_{g b e s t}$ is globally optimal solution. $C$ is the swimming step, $P(x, y)$ is the position of bacteria. This calculation is performed in order to improve analysis of the convergence speed.

\section{Copy optimization operation.}

In order to improve group information sharing mechanism in bacterial foraging algorithm and enhance global search performance of the algorithm, probability density function of individual bacterium under quantum space is established by utilizing shared information of bacteria at the copy stage, and position of different individual bacteria is updated through Monte Carlo random sampling. Thus copy operation of the population is realized, and loss of population diversity caused by direct copy is avoided. In this way, quantum bacterial foraging optimization (QBFO) algorithm is formed. In QBFO, a quantized potential well is required to restrain individuals and endow the population with aggregation state. Bacteria under quantum bound state can appear at any point in the space at a certain probability density, and the probability tends to be 0 when the distance of particles and the center is infinite. Suppose in a $D$-dimensional search space, at iteration $t$, the position of individual $i$ is: $x_{i}(t)=\left(x_{i 1}(t), x_{i 2}(t), x_{i d}(t) \ldots x_{i D}(t)\right)$, the best position of the individual is $p_{i}(t)=\left(p_{i 1}(t), p_{i 2}(t), p_{i d}(t) \ldots p_{i D}(t)\right) ;$ and the best position of the overall situation is $p_{g}(t)=\left(p_{g 1}(t), p_{g 2}(t), p_{g d}(t) \ldots p_{g D}(t)\right)$. According to individual particle track analysis, in order to guarantee convergence of the algorithm, individual must converge in the local convergence point $p_{i}=\left(p_{i 1}, p_{i 2}, . . p_{i d} \ldots p_{i D}\right)$; the local convergence point $p_{d}$ of each bacterium $i$ at each dimension should meet:

$$
p_{d}(t)=\left(\varphi_{1 d} p_{i d}(t)+\varphi_{2 d} p_{g d}(t)\right) /\left(\varphi_{1 d}+\varphi_{2 d}\right)
$$

Where $\varphi_{1 d}$ and $\varphi_{2 d}$ are random data in $(0,1)$. In quantum space, state and position of individual bacterium are uncertain and have to be determined by the wave function $\psi(Y, t) .|\psi|^{2}$ is the probability density function of individual position. An attractive potential based on Delta potential model is established at each dimension of the attractor, and the potential-energy function can be gained:

$$
V(Y)=-\gamma^{*} \delta(Y)
$$

Where $Y=x_{i d}-p_{d}$ indicates the distance between individual position $x_{i d}$ and the attractor. By substituting it into quantum mechanics equation, wave function and probability density function of each dimension can be obtained: 


$$
\begin{aligned}
& \psi(Y)=\frac{1}{\sqrt{L}} e^{-|Y| / L} \\
& Q(Y)=|\psi(Y)|^{2}=\frac{1}{L} e^{-2\left|x_{i d}-p_{d}\right| / L}
\end{aligned}
$$

In potential well, position of the individual bacterium obeys the above probability density function. In order to determine the position of any individual bacterium at any certain time, individual bacterium equation after iteration for $t+1$ times is acquired on the basis of Monte Carlo simulation, and the position update equation of bacterium $i$ at $d$ dimension is determined:

$$
\begin{aligned}
& x_{\text {id }}(t+1)=p_{d}(t) \pm \frac{L}{2} \ln (1 / u), u \in U(0,1) \\
& L=2 \alpha \cdot\left|C(t)-x_{\text {id }}(t)\right|
\end{aligned}
$$

Where $u$ is random data in $(0,1)$; in this paper, $u=e^{-\left|x_{i d}-p_{d}\right| / L} \cdot C(t)$ means the average of the best position vectors of the population. The average optimal position is presented in formula (10).

$$
C(t)=\sum_{i=1}^{N} p_{i}(t)
$$

$\alpha$ is the contraction and expansion coefficient, $\alpha<1.782$; it meets the following three linear, convex and concave expressions[13]:

$$
\alpha(t)=\left(\alpha_{0}-\alpha_{1}\right)\left(t / t_{\max }\right)^{2}+\left(\alpha_{1}-\alpha_{0}\right)\left(2 t / t_{\max }\right)+\alpha_{0}
$$

In which with linear transformation of evolution algebra $t$ from $\alpha_{0}$ to $\alpha_{1}, t_{\max }$ is the maximum iteration time. According to QBFO update equation, each dimension variable $x_{i d}$ obeys probability distribution centering on attractor $p_{d}$ in the range of $L$ during the update process of bacteria position. Compared with traditional evolutionary algorithm, QBFO has more powerful randomness and stronger global search ability by combining statistical theory with random optimization.

\section{Implementation Process of Ship Collision Avoidance}

Application of QBFO algorithm in ship collision avoidance path optimization[9][10][11][12] involves two aspects which are problem solution encoding and objective function establishment.

\section{Encoding of collision avoidance problem}

The model of ship collision avoidance path planning is used to solve the four parameters including $t_{s}, t_{a}, \Delta C_{0}, \Delta C_{b}$ :

$t_{\text {collision }}$ is the time when the distance between our ship and the target ship is the shortest when our ship moves along the original route. In order to guarantee safety of the ship, steering collision avoidance must be conducted before $t_{\text {collision }}$. $\left(0<t_{s}<t_{\text {collision }}\right)$.

$t_{a}$ is the sailing time after collision avoidance ,data range of it should be diminished to enhance the search efficiency, which means $t_{s}+t_{a} \geq 3^{*} t_{\text {collision }}$, and it should equal the new DCPA $t_{C P A 1}$ after collision avoidance behavior is adopted. The calculation process for $t_{a}$ is determined according to the actual simulation, as shown in the following: 


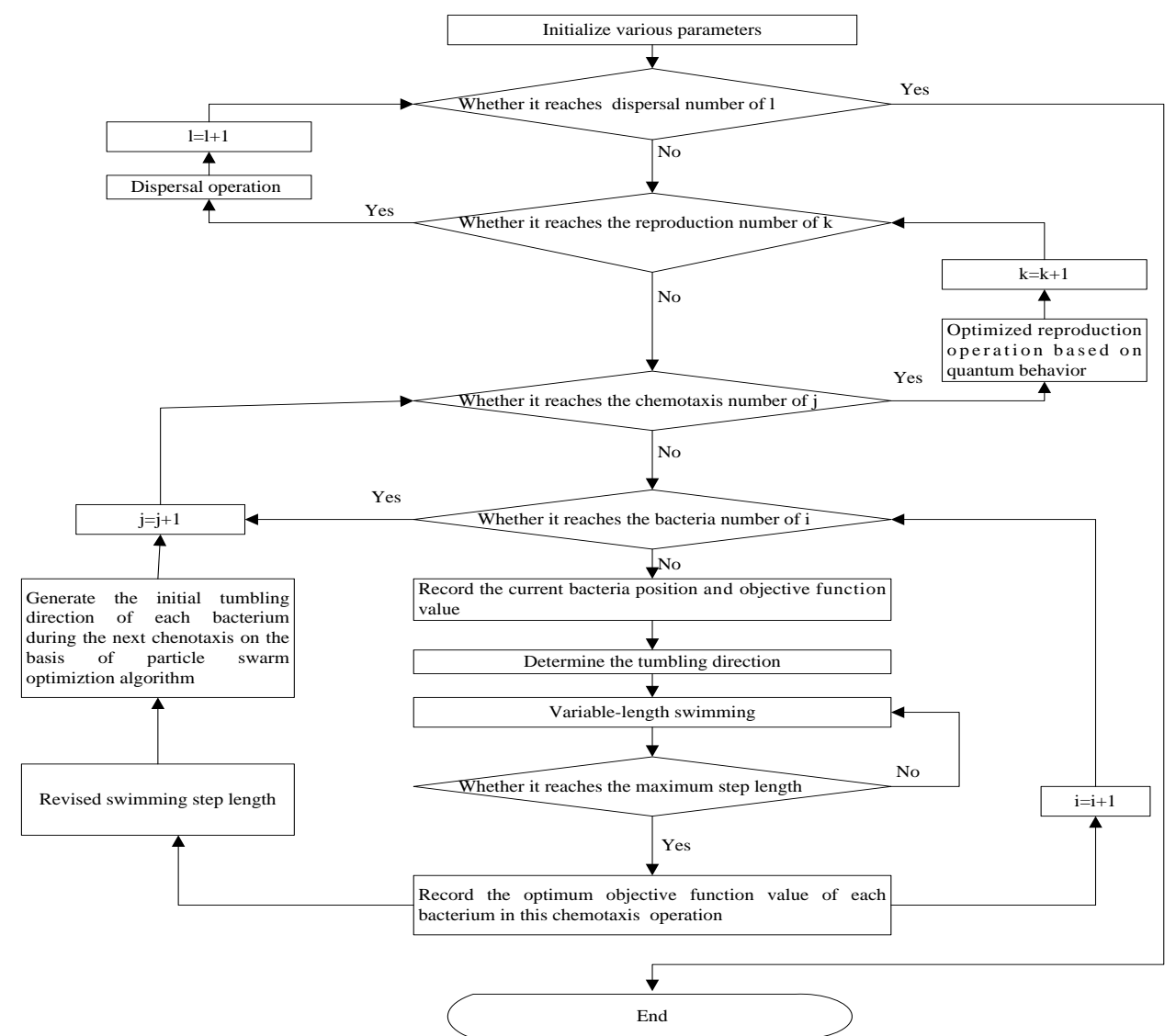

Fig. 3. Optimized bacteria forging algorithm

(1) If $d_{C P A 1}<d_{G}$, then ${ }^{t_{a}}=40 \mathrm{~min}$; thus, the objective function is too large, and the optimization process is directly eliminated.

(2) Under the condition $d_{C P A 1} \geq d_{G}$, if $t_{C P A 1}>0$, then $t_{C P A 1}=t_{a}$ is the starting point. If $d_{C P A 2} \geq d_{G}$ is not satisfied, then $t_{a}$ will increase by a certain step value.

(3) Under the condition $d_{C P A 1} \geq d_{G}$, if $t_{C P A 1}<0$, then the initial value $t_{a}=0.05 * 60$; if $d_{C P A 2} \geq d_{G}$ is not satisfied, then $t_{a}$ will increase by a certain step value (the search step value adopted by this paper's simulation is equal to $0.02 * 60$ ).

Where, the value of $d_{G}$ is determined by the zero boundary of the space risk index.

$\Delta C_{0}$ and $\Delta C_{b}$ are angle at the time of collision avoidance (direction), and angle at time of resumption (direction);

Based on the above analysis, in order to accelerate operation of the algorithm, ship collision avoidance path planning decision-making based on quantum bacterial foraging algorithm can be treated as encoding of 2 parametric variables. As for the individual bacterium $i, \beta_{i}=\left[\Delta C_{0}, \Delta C_{b}\right]$.

\section{Establishment for objective function of vessel collision avoidance}

In the process of planning a collision avoidance path, safe collision avoidance should be guaranteed first and then "economical" collision avoidance may be considered. The collision avoidance optimization algorithm is required to complete collision avoidance along the shortest path with the premise of ensuring safety; the objective function is as follows:

$$
J=\min \left(d_{s}+d_{r}\right)
$$

Where, $d_{s}$ is the sailing distance of the ship after collision avoidance, and $d_{r}$ is the sailing distance of the ship at the resumption stage. Suppose that velocity of the ship is $V$, the steering 
angle of avoidance is $\Delta C_{0}$, the steering angle at the resumption stage is $\Delta C_{b}$, and the sailing time after avoidance is $t_{a}$. Therefore, $d_{s}$ and $d_{r}$ in the above objective function meet the following expression:

$$
\begin{aligned}
& d_{s}=t_{a} * V \\
& d_{r}=t_{a} * V * \sin \Delta C_{0} /\left|\sin \Delta C_{b}\right|
\end{aligned}
$$

In the process of path planning, a constraint condition should be provided for the variables in Formula (15) and (16), as shown in Formula (4)

$$
\begin{aligned}
& 30^{\circ} \leq \Delta C_{0} \leq 90^{\circ} \\
& -60^{\circ} \leq \Delta C_{b} \leq-30^{\circ} \\
& t_{C P A 1} \leq t_{a} \leq 40 \mathrm{~min} \\
& d_{C P A 1} \geq d_{G}, d_{C P A 2} \geq d_{G}
\end{aligned}
$$

Where $d_{C P A 1}$ and $d_{C P A 2}$ are the new DCPA after collision avoidance and course restoration, and the value of $d_{G}$ is determined by the zero boundary of the space risk index. The sailing time after collision avoidance is ${ }^{t}$, and it should equal the new DCPA $t_{C P A 1}$ after collision avoidance behavior is adopted.

\section{Implementation Steps of Ship Collision Avoidance Path Planning Algorithm}

Step 1: Parameter determination[14] in the algorithm implementation process includes as in search space dimension of bacterial foraging algorithm $P=2$; bacterial quantity used for search $S=26$; and number of convergence $N_{c}=50$; the maximum swimming step of movement is required to be 0.5 and the minimum swimming step of movement is required to be 0.05 , which can guarantee a good search effect as in[15], and the number of replication $N_{r e}=4$; the number of migration $N_{e d}=2$; value range of migration possibility should be $0.05-0.3$, and it is $P_{e d}=0.25$ in this paper; In particle swarm optimization, $C_{1}, C_{2}, R_{1}$ and $R_{2}$ are random parameters. And the maxi-mun and mini-mun of contraction and expansion coefficient are following $\alpha_{0}=0.8, \alpha_{1}=0.6$, finally, the maximum number of iterations is $N_{c} \times N_{r e} \times N_{e d}=400$;

Step 2: Start the circulation process; migration operation $l=N_{e d}$; copy operation $k=N_{r e}$; chemotactic operation $j=1: N_{c}$; start nested loop;Step3:Initialize the population, randomly generate $S$ independent two-dimensional individual bacterium parameters $P\left(P_{1}, P_{2}\right)$ in the solution space, and calculate the initial objective function value $J$ of various bacteria;

Step 4: Execute the process of chemotactic operation circulation; execute flipping and movement operation for each bacterium $i$ in the two-dimensional space to generate the random new position $P\left(P_{1}, P_{2}\right)$ of bacteria;

$$
P_{2}=P_{2}+C(i, j, w) * \text { Delta/sqrt(cov) } \quad P_{1}=P_{1}+C(i, j, w) * \text { Delta } / \operatorname{sqrt}(\mathrm{cov})
$$

Where Delta $=2 *(($ double $)$ rand ()$) /$ RAND_MAX $-1.0 ; \operatorname{cov}+=\operatorname{pow}($ Delta, 2.0$)$ means the flipping direction of bacteria. Update the chemotactic movement step length of each bacterium in one chemotactic operation according to formula 1.

Step 5: Record the optimal objective function when all bacteria complete one chemotactic operation; find out the objective function value of particles corresponding to the global optimal solution $P_{g b e s t}$ and single optimal solution $P_{\text {lbest }}$ after this chemotactic circulation. Update the particle state as per particle swarm optimization and select the new direction and position of the 
next chemotactic operation according to (2) and (3). Execute step 6 after completing all chemotactic operations; otherwise, turn to step 3.

Step6: After all bacteria pass the complete chemotactic operation based on bacteria reproduction operation of quantum behavior, acquire the best position and global optimal position of different bacteria in the population. After calculating the best vector, update the population position according to formula (8).

Step7: Execute migration operation for all bacteria with low fitness after copy operation according to the migration probability, and acquire the random new position in two-dimensional space.

Step8: Make a judgment after the circulation and output the two-dimensional results of the optimal avoidance angle and resumption angle.

\section{Simulation Test and analysis}

Dynamic ship collision avoidance simulation is realized via an intelligent bacterial foraging algorithm of particle swarm under the interface of VC++9.0 environment based on VS2010 . Take the example of head-on-head approach of a single ship , the initial motion parameters of our ship are: $V=13, B=0^{\circ}$ and the coordinate is $(0,0)$; the initial motion parameters of the target ship are: $V=14, B=180^{\circ}$ and the coordinate is $(0,4)$. According to collision risk index analysis, CRI=1, $\mathrm{DCPA}=0$ and $\mathrm{TCPA}=-0.15$. There is collision risk at the preliminary stage, and the collision avoidance process is implemented directly, as shown in Fig. 4.

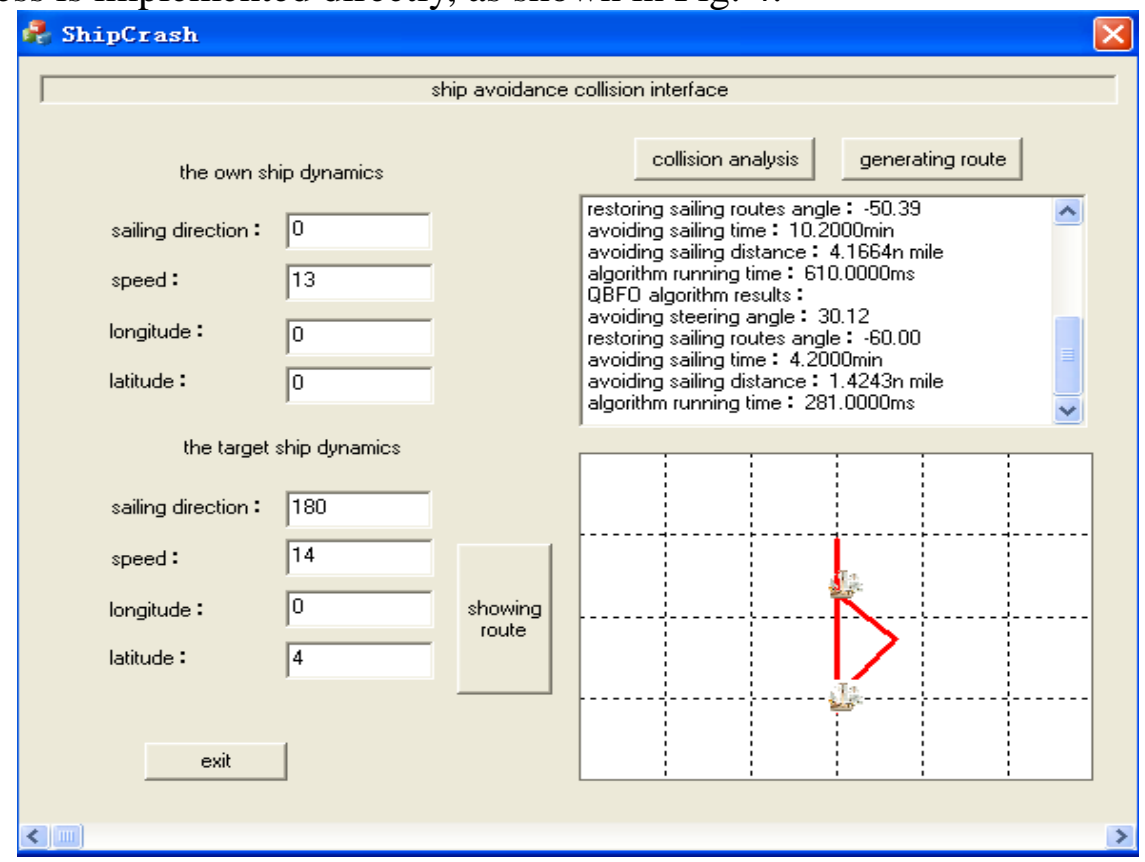

Fig.4. the interface of head-on ship avoidance collision

According to the final optimization result, an optimal collision avoidance strategy is selected when the rudder angle is 30.12 degrees, according to the fitness priority. the distance of two vessels is smaller and smaller with the navigation. The DCPA has a jump from Time 2.6 min when the collision avoidance operation is adopted. 


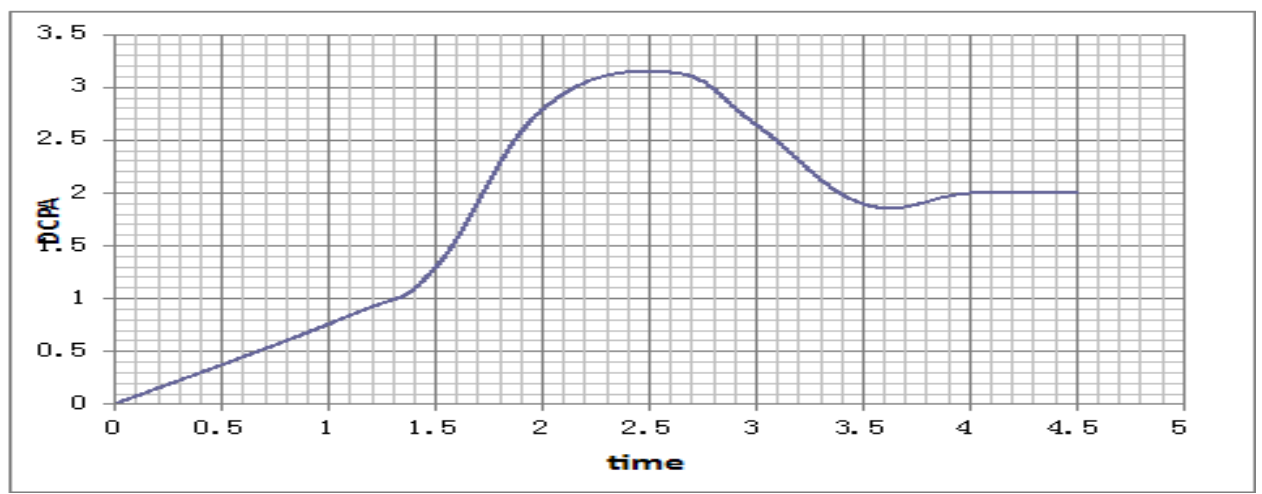

Fig.5. the change of DCPA with the time

In the above collision avoidance interfaces, clicking the path generation buttons presents the comparison between the BFO algorithm and the QBFO algorithm in optimized collision avoidance parameters, as shown in Table 1. On the one hand, the QBFO algorithm can reduce the ship avoidance angle, reasonably select the resumption angle for the ship, decrease the sailing time of avoidance, and shorten the sailing distance-all of this contributes to greater efficiency in intelligent ship avoidance. Meanwhile, the QBFO algorithm can increase the convergence rate and reproduction quality of bacteria, so that the time taken by the algorithm is decreased. This provides greater collision avoidance time for seaman and reduces the possibility of improper operation.

Table 1 Optimized Collision Avoidance Parameters

\begin{tabular}{|l|c|c|c|l|l|}
\hline & $\begin{array}{l}\text { Avoiding steering } \\
\text { angle }{ }^{\circ}{ }^{\circ}\end{array}$ & $\begin{array}{l}\text { Resumption } \\
\text { sailing routes } \\
\text { angle }\left({ }^{\circ}\right)\end{array}$ & $\begin{array}{l}\text { Avoiding } \\
\text { sailing time } \\
(\text { min) }\end{array}$ & $\begin{array}{l}\text { Avoiding sailing } \\
\text { distance } \\
(\mathrm{n} \text { mile })\end{array}$ & $\begin{array}{l}\text { Algorithm } \\
\text { running time } \\
(\mathrm{ms})\end{array}$ \\
\hline BFO head-on & 43 & -50.39 & 10.2 & 4.1664 & 610 \\
\hline $\begin{array}{l}\text { QBFO } \\
\text { head-on }\end{array}$ & 30.12 & -60 & 4.2 & 1.4243 & 280 \\
\hline
\end{tabular}

\section{Conclusion}

This paper applies QBFO algorithm to ship collision avoidance and path optimization.First,the PSO algorithm is applied to the BFO to optimized the bacterial tumbling direction and the new position for the next chemo taxis to improve the local convergence rate; Second, the adaptive sliding step which deceases linearly is adopted to strengthen the convergence ability of local search; Thirdly,the bacteria individual is described in the quantum space and a potential well model is created. Using Monte Carlo method to achieve the reproduction of bacterial reproduction , and which makes the population are able to search the whole space. then,the objective function based on the shortest collision avoidance path is established to optimize the avoidance and steering angle. Finally, the dynamic collision avoidance process, based on the QBFO algorithm applied in situations of ship encounter, is demonstrated via the VS2010 simulation interface, which displays the comparison parameters of collision avoidance and verifies directly and visually the feasibility and superiority of applying this algorithm to intelligent ship collision avoidance. This research lays a foundation for the next step of the implementation of a multi-ship collision avoidance process.

\section{Acknowledgment}

It is appreciated that this research is subsidized by funding from the Fundamental Research Funds for Central universities. (No. HEUCFX41305)

\section{References}

[1]Lee, S. M., Kwon, K. Y. and Joh, J. A fuzzy logic for autonomous navigation of marine vehicles satisfying COLREG guidelines[J]. International. Journal Of Control Automation And Systems, 
2004,2, 171-181.

[2]Statheros, T., Howells, G. and McDonald-Maier K. Autonomous ship collision avoidance navigation concepts, technologies and techniques[J]. The Journal of Navigation, 2008. 61:129-142.

[3]Smierzchalski, R. and Michalewicz, Z.. Modeling of ship trajectory in collision situations by an evolutionary algorithm[J]. IEEE Transactions On Evolutionary Computation, 2000,4:227-241.

[4]Tsou Ming-Cheng;Hsueh,Chao-Kuang. The Study of Ship Collision Avoidance Route Planning by Ant Colony Algorithm.Journal of Marine Science and Technology-Taiwan. 2010.18(5):746-756.

[5] Cockcroft, A. N. and Lameijer, J. N. F. (2011). A Guide to Collision Avoidance Rules. Butterworth-Heinemann.

[6]COLREGS. (1972) ([with amendments adopted from December 2009]). Convention Convention on the International Regulations for Preventing Collisions at Sea.International Maritime Organization, London.

[7]H.Supriyono, M.O.Tokhi. Bacterial Foraging Algorithm with Adaptable Chemotactic Step Size[C]. Second International Conference on Computational Intelligence, Communication Systems and Networks.2010,72-77.

[8]JiangJianguo,ZhouJiawei,ZhangYingchun.Adaptive Bacterial Foraging Optimization Algorithm [J].journal of Xian university. 2015,42(1):75-80.DOI:10.3969/j.issn.1001-2400.2015.01.012.

[9]Wangkun,Zhanghongyang,Liuzhi. Path Planning for Robots Based on Quantum-behaved Particle Swarm Optimization[J].2010,26(4):155-157.

[10] Sun J, Fang W, Palade V. Quantum-behaved Particle Swarm Optimization with Gaussian Distributed local attractor point [J]. Applied Mathematics and computation.2011,218(7):3763-3775.

[11]FangWei, Sun Jun, Xie Zhenping. Convergence analysis of quantum-behaved particle swarm optimization algorithm and study on its control parameter [J]. Acta Physica Sinica, 2010, 59(6):3686-3694.

[12]Zhangguoyong,Wuyonggang,Tanyuxiang. Bacterial Foraging Optimization Algorithm with Quantum behavior. Journal of Electronics \& information technology. 2013, 35(3): 614-620.

[13] Fang Wei,Sun Jun,Xie Zhen- Ping,Xu Wen- Bo.Convergence analysis of quantum- behaved particle swarm optimization algorithm and study on its control parameter.[J].Acta Physical Scinica, 2010,59 (6): :3686-3694

[14]Ma Wenyao,Yang Jiaxuan.Optimization of ship collision avoidance route based on bacterial foraging algorithm.Journal of Dalian Maritime University[J]. 2013,39(2): 21-24.

[15]M.D.Nguyen,V.T.Nguyen,H.Tamaru.Automatic Collision Avoiding Support System for Ships in Congested Waters and at Open Sea. International conference on Control, Automation and Information Sciences.2010.96-101, DOI: 10.1109/ICCAIS.2012.6466638 